\title{
Criminologie
}

\section{Les paradigmes du droit pénal et les criminologies cliniques}

\section{Christian Debuyst}

Volume 25, numéro 2, 1992

Nouvelles connaissances et nouvelles questions en criminologie

URI : https://id.erudit.org/iderudit/017322ar

DOI : https://doi.org/10.7202/017322ar

Aller au sommaire du numéro

Éditeur(s)

Les Presses de l'Université de Montréal

ISSN

0316-0041 (imprimé)

1492-1367 (numérique)

Découvrir la revue

Citer cet article

Debuyst, C. (1992). Les paradigmes du droit pénal et les criminologies cliniques. Criminologie, 25(2), 49-72. https://doi.org/10.7202/017322ar

\section{Résumé de l'article}

This paper explores the relations between different clinical perspectives in criminology, and the current criminal law. The author identifies the existence of at least two major perspectives. The predominant approach is well-integrated with the existing criminal law. A second, less widespread perspective is incompatible with the autoritarian discourse of existing criminal law. This perspective stresses the need to undertake a paradigm shift in terms of the criminal law. 
This paper explores the relations between different clinical perspectives in criminology, and the current criminal law. The author identifies the existence of at least two major perspectives. The predominant approach is well-integrated with the existing criminal law. A second, less widespread perspective is incompatible with the autoritarian discourse of existing criminal law. This perspective stresses the need to undertake a paradigm shift in terms of the criminal law.

Le thème que nous voudrions aborder nous renvoie autant à la recherche qu'à la pratique telle qu'elle se déroule au niveau du tribunal ou dans le cadre de l'exécution d'une mesure: établissement pénitentiaire ou établissement pour jeunes ${ }^{2}$. On pourrait le résumer de la manière suivante: quel rapport existe-t-il entre le type d'attitude que l'on prend en clinique criminologique et le cadre penal dans lequel cette attitude prend place? D'une manière plus précise, nous voudrions décrire deux attitudes possibles (qui nous renvoie à deux types de criminologie) et démontrer qu'elles s'intègrent différemment dans le cadre pénal tel qu'il existe (tant au niveau des adultes que des mineurs). Quelles conséquences en tirer? Ne faut-il pas se poser la question à savoir si un changement de paradigmes à ces deux niveaux (pénal et criminologique) ne s'avère pas nécessaire?

\section{LES PERSPECTIVES CLINIQUES ET LEURS POSSIBILITÉS D'INTÉGRATION DANS LE SYSTĖME PÉNAL}

\subsection{Les deux orientations cliniques dans la prise en compte de la délinquance et de son auteur}

Nous pourrions résumer les deux orientations de la manière suivante.

La première orientation. On peut considérer que la délinquance nous renvoie à un problème général à propos duquel nous chercherions à mettre au point, pour l'expliquer, une théorie générale qui soit valable quelles que soient les formes de délinquance. La délinquance est une

1. Professeur, Université Catholique de Louvain, 1, Place de l'Universitê, 1348 Louvain-la-Neuve, Belgique.

2. Cet article reprend et prolonge l'intervention faite au département de criminologie de l'Université d'Ottawa en mars 1991. Voir Debuyst (1991). 
transgression de la règle pénale, et le fait de transgresser la loi permet de considérer son auteur comme délinquant et de lui attribuer un statut qui implique que l'on puisse prononcer à son égard une peine ou une mesure de traitement (la notion de traitement étant entendue dans son sens large). En d'autres termes, le sujet devient «objet» d'une attention particulière qui répond à la «faille» comportementale qui explique sa conduite. On peut ici se reporter à une explication générale qui serait valable quelles que soient les formes de délinquance et qui traduirait essentiellement une certaine "insensibilité » à la loi et à la sanction. Elle serait psychologique ou psychosociale et pourrait être qualifiée de «degré d'engagement dans la délinquance" (Hirschi), ou de "caractère d'antisocialité» (Jesness) ou encore de «personnalité criminelle» (Pinatel, LeBlanc, Favard, etc.). On peut dire que, selon cette optique, tous les individus se situeraient à un endroit déterminé de continuum qui traduirait le degré d'engagement dans la délinquance du sujet. Il en résulte qu'il ne serait pas tellement intéressant de connaître ou de tenir compte du type d'infraction, ni de ce que la délinquance se déroule en groupe ou pas, que les délinquants appartiennent à telle ou telle classe sociale. L'important serait de dégager la variable en question au-delà des différentes formes à travers lesquelles elle s'exprime. On voit dans ces conditions ce que constitue l'examen médico-psychologique et social. II portera essentiellement sur le sujet qui est « objet de préoccupation ». Dans cette hypothèse, la criminologie se définira comme «étude scientifique du crime et du délinquant», et une accumulation de connaissances peuvent se mettre en place dans ce but. Cette orientation a été dominante dans la criminologie clinique jusqu'à présent et se caractérise donc, entre autres, par le fait de voir la délinquance comme une «faille».

La deuxième orientation se traduit par une complexification du point de départ: elle nous amène à définir la délinquance non tellement comme une infraction mais comme constituée par des comportements qui se déroulent dans des situations nettement circonscrites et qui prennent place dans un contexte psychologique, social, politique également nettement circonscrit qu'il importe d'aborder comme tel et où se manifestent des interrelations ayant aussi une certaine spécificité. Cela veut dire que la délinquance, d'abord nous renvoie à des situations qu'il importerait de traiter comme telles ${ }^{3}$ : si elle prend place et sens dans

3. Ainsi, un vol dans les grands magasins n'est pas la même chose qu'un holdup, même s'il existe cet élément commun que constitue la transgression de la loi pénale. Non seulement le fait differe, mais également le contexte interrelationnel dans lequel il se déroule. Ce sera à travers ce contexte que les règles prennent sens ainsi que le comportement du sujet. 
l'histoire d'un sujet, elle se situe comme moment dans une relation conflictuelle qui met non seulement aux prises des individus, mais aussi ce sujet et le groupe social (ou certaines entités constitutives de ce groupe social) en tant qu'il est instaurateur de règles. La définition de la criminologie que nous serons amené à donner introduira comme objet, en plus du crime et du délinquant, le groupe en tant qu'il instaure les règles dans un grand nombre de situations et qu'il les applique. À ce point de vue, il ne faut pas oublier que les instances légales et judiciaires ne sont pas les seules qui soient instauratrices de règles. On doit également noter les instances familiales, scolaires, professionnelles, etc. Même si elles occupent un statut particulier, nous n'avons pas de raison majeure d'isoler les instances pénales. Néanmoins, comme nous parlons de délinquance et que nous nous situons en criminologie, ce sera jusqu'à nouvel ordre à travers les «situations pénales» que ces différents milieux instaurateurs de règles seront pris en compte.

Une difficulté importante se pose néanmoins: cette deuxième orientation s'inscrit peu ou mal dans le droit pénal (que ce soit celui qui concerne les majeurs ou les mineurs) tel qu'il existe, alors que ce n'est pas vrai pour la première. En effet, comme celle-ci prend en compte seulement la délinquance comme comportement et les caractéristiques individuelles susceptibles de l'expliquer, elle trouve dans le paradigme pénal actuel un cadre dans lequel les informations qu'elle donne s'inscrivent assez naturellement. Ce serait à voir de plus près.

\subsection{Une criminologie clinique axée sur une définition de la délinquance comme *faille» de la sociabilité: sa parfaite intégration dans le droit pénal}

Il est cependant nécessaire de faire une mise au point. On a dit, avec raison, que la criminologie clinique qui voit la délinquance comme " faille " s'était constituée au début en opposition avec le droit pénal (classique). Dès lors, comment peut-elle s'y inscrire aussi facilement ? Pour répondre à cette question, et si conflit il y a, il importe de préciser le lieu où se situe cet éventuel conflit, car, lorsqu'on parle du droit pénal comme cadre de la criminologie, le terme de cadre doit être envisage dans au moins trois sens: $a$ ) on peut dire qu'il est cadre dans le sens où il nous indique les types de comportements définis comme infraction; $b$ ) il l'est également en tant qu'il définit le statut du sujet qui aurait commis une infraction, c'est-à-dire celui de devenir «objet », que ce soit objet de punition ou objet d'une mesure quelconque; et finalement $c$ ) il constitue un cadre dans lequel se définit le type de mesure pénale et la manière dont celle-ci doit être organisée: autour de la notion de responsabilité et de 
punition, ou autour de celle de déterminisme et de défense sociale, avec toutes les nuances que nous pourrions introduire.

Pour une criminologie axée sur la délinquance comme « faille», on peut dire que les points " $a$ » et " $b$ » sont des $a$ priori qu'elle accepte comme points de départ, et, en ce sens, le droit pénal est effectivement le cadre de cette criminologie. Mais ce que ces criminologues n'acceptent pas est la manière dont le droit pénal organise la mesure pénale, $c$ 'est-à-dire ce qui touche au point « $c$ ». Ils affirment en effet la nécessité d'organiser cette réaction pénale autrement que par la manière du droit pénal classique ou néo-classique et de le faire à partir d'une «connaissance scientifique» du délinquant et aussi d'une analyse des résultats obtenus par les mesures déjà appliquées.

Dire qu'il faut partir d'une "connaissance scientifique» pour organiser la mesure penale signifie que, dans cette perspective, la règle fondamentale qui s'imposera à toute intervention médio-psychosociale sera celle de ce qu' «il importe de connaître avant d'intervenir ». C'està-dire que toute mesure exige comme préalable la mise en place d'un examen médico-psychologique et social qui visera à pouvoir répondre adéquatement aux questions du traitement qui, généralement, s'organisent autour de deux pôles: la dangerosité du sujet et ses possibilités de réinsertion ${ }^{4}$. Toute l'histoire de cette criminologie clinique dominante est marquée par cette préoccupation. Elle est affirmée par Lombroso: «On doit traiter les criminels individuellement, modifier le traitement suivant le caractère personnel, si l'on veut obtenir un résultat quelque peu satisfaisant. Comment pourrait-on pratiquer la libération conditionnelle, administrer avec succès une maison de correction, sans étudier individuellement le crime ${ }^{5}$ " J. Pinatel rappelle que cette position fut reprise notamment par le $\mathrm{D}^{\mathrm{r}}$ Vervaeck, le psychiatre belge qui créa, en 1917, le service anthropologique dans le cadre des prisons: « on ne peut songer, sans étude scientifique adéquate, méthodique et approfondie de chaque cas, à fixer un traitement rationnel et efficace de la délinquance ${ }^{6}{ }_{\text {» }}$ Nous retrouvons cette affirmation tout au long des années et, dans la documentation la plus actuelle, nous la voyons explicitement réaffirmée?

4. Cette question ne se posera pas nécessairement avant le jugement, mais au moins à partir du moment où commencera le traiternent, qu'il s'agisse d'un traitement pénitentiaire ou d'un traitement prenant une autre forme. Il s'agit la d'une question cle a propos de laquelle des ententes peuvent facilement se faire entre pênalistes et criminologues.

5. Cité par Pinatel $(1963$, p. 396).

6. Pinatel (1963, p. 399).

7. Nous pourrions e.a. faire allusion, au Québec, à M. LeBlanc et, en France, a A. M. Favard (voir Cario et Favard, 1991). 
À ce propos, la criminologie s'est définie non seulement comme science du crime et du délinquant, mais aussi comme «étude scientifique des peines" afin de rechercher, «d'une manière expérimentale», comme le disait E. Ferri, si elle atteignait leur objectif ${ }^{8}$. Et c'est d'ailleurs à ce niveau que s'est ouvert le débat entre la criminologie naissante et le droit pénal. Il porte sur la manière dont le sujet délinquant doit être pris comme «objet»: objet de punition ou objet d'une mesure de défense sociale. Un tel débat n'est nullement négligeable et concerne, outre un problème d'efficacité, une manière autre de poser le statut de la personne envisagée dans ces deux «systèmes» de réaction pénale ${ }^{9}$.

On doit néanmoins reconnaître que, si conflit il y a, entre le droit pénal classique et néo-classique et la criminologie, celui-ci est nettement localisé et que d'autres points sont communs. Il existe en effet entre les deux perspectives un type de langage qui fonctionne dans un cadre autoritaire: du fait de la reconnaissance de certains comportements comme infractions, leur auteur devient objet de punition ou objet de traitement.

\subsection{La difficulté d'intégrer la deuxième orientation dans le droit pénal}

Ce cadre autoritaire rend en effet impossible de prendre en compte ce qu'implique une orientation qui situerait le sujet, comme nous l'avons dit, dans une situation complexe liée aux contextes dans lesquels les infractions se déroulent et à la manière dont les interdictions se constituent, s'imposent et sont vécues. Un cadre autoritaire, en effet, nous renvoie à des instances évaluatrices pour lesquelles l'interrelation est dominée par la réduction de l'une des parties au comportement de transgression qu'elle a commise et dans lequel elle se trouve en quelque sorte «figé " ${ }^{10}$. Mais s' affirment, dans cette même situation et comme en réponse, la réaction de celui qui se trouve mis sous un tel regard, les justifications qu'il donne et se donne, les arguments sur lesquels de

8. Voir plus particulièrement les texte d'E. Ferri. À ce propos: Debuyst C. (1990).

9. Le système axé sur le libre arbitre et la notion de peine donnent aux justiciables un certain nombre de garanties, alors que le système axé sur les mesures de défense sociale est capable de tenir compte des difficultés que pose la situation rélle dans laquelle se trouve le sujet. Il suffit, pour en prendre conscience, de se référer à la documentation des positivistes italiens. Il faut être attentif au fait qu'existe, en Belgique, une loi de Défense sociale (1930) qui règle la situation des délinquants anormaux. C'est a cette loi que nous nous reportons lorsque nous parlons, dans cet article, de mesures de défense sociale.

10. Voir Debuyst (1985a). 
telles justifications peuvent effectivement se soutenir. Dans une perspective autoritaire, ces données ne sont pas prises en compte, ou le sont mal (nous le verrons ultérieurement). Elles le seraient dans la deuxième orientation qui, de ce fait, ouvrirait un autre débat et permettrait une solution qui soit à la fois non réductrice et qui tienne compte du caractère problématique que la transgression pose au groupe social lui-même et qui, d'ailleurs, variera selon le type d'infraction commise. C'est donc dire que, même s'il y a transgression, ce qui importe est la problématique interrelationnelle qui se noue et qui s'est nouee, et dans laquelle les parties mises en cause apparaissent comme acteurs, c'est-à-dire comme des être réfléchissant et agissant en fonction de nombreuses variables. Celles-ci influent sur les interdits tels qu'ils sont exprimés et vécus, sur le comportement du groupe social ainsi que sur celui du sujet délinquant. Il en résulte que, dans la première orientation, les connaissances qu'il importe d'acquérir se situent autour de ce qui concerne l'examen médico-psychologique et social de la personnalité du sujet et les facteurs qui l'ont influencé. Dans la deuxième, cette nécessité de connaissances se situerait autour d'une analyse des interrelations qui se déroulent à propos des confrontations que vit le sujet à l'intérieur du contexte d'ensemble ainsi que du caractère plus ou moins menaçant des ruptures que suscite, pour le délinquant, une affirmation de soi comme pour le groupe social, une affirmation de la loi.

Cette deuxième manière de poser le problème s'inscrit peu ou mal dans le droit pénal tel qu'il existe: les variables mises en cause ne se localisent plus seulement sur l'auteur de l'acte, mais aussi sur la responsabilité du groupe social; et il importerait, de ce fait, de donner à cet "auteur», comme aux autres acteurs, un statut que le caractère autoritaire du droit pénal ne lui permet pas de donner. Lorsque nous utilisons les termes fort à la mode de «responsabilité» et de «responsabilisation », il ne suffit pas de penser exclusivement au sujet «délinquant »; la prise en compte de la pluralité des acteurs nous paraît primordiale.

\section{LE CARACTĖRE AUTORITAIRE DU DISCOURS CRIMINOLOGIQUE DOMINANT ET LES EMBARRAS QUI EN RÉSULTENT}

Face à cette situation, quelles sont les difficultés qui nous empêchent d'intégrer les éléments qui participent à cette deuxième orientation : c'est le fait, comme nous l'avons vu, que le discours pénal comme le discours criminologique (tel qu'il s'exprime dans la première orientation) sont des discours autoritaires et que, dès l'abord, ils s'imposent au 
discours individuel et voilent ce que celui-ci pourrait présenter d' «objectif ».

\subsection{La notion de discours autoritaire de Boltanski}

La question qui se pose dès lors est celle de savoir comment, dans quelle mesure et à quel niveau le protagoniste « délinquant » est capable de faire valoir ses justifications.

D'abord, il faut s'entendre sur ce qu'on entend par « discours autoritaire". La manière dont nous posons la question découle des analyses faites par L. Boltanski ${ }^{11}$ sur le caractère plus ou moins fort que présentent, à propos d'un événement déterminé, le discours ou les justifications des divers protagonistes. Ainsi, pour reprendre nos exemples, l'interprétation que donne le pénaliste ou le scientifique (criminologue qui, par exemple, détermine la dangerosité) et qu'ils opposent à celle qu'en donne l'individu tend assez naturellement à l'emporter: elle constitue une interprétation forte non seulement parce qu'elle émane de la part de quelqu'un qui a un pouvoir de décision, mais également parce qu'au niveau d'une certaine rationalité elle a une cohérence interne; elle se réfère à un système interprétatif reconnu et faisant l'objet d'un certain consensus face auquel le discours du sujet apparaît comme étant d'un poids faible, d'autant plus que l'objectif poursuivi lui échappe partiellement. Cela nous paraît aussi vrai pour le diagnostic scientifique (psychiatrique ou psychologique) que pour la décision judiciaire. Les deux émanent de personnes qui disposent d'un pouvoir ainsi que d'une grille de lecture dont la rationalité et les justifications sont jugées, à priori, supérieures. La question qui se pose est celle de savoir comment infléchir ce caractère «autoritaire » pour rendre recevable le discours du sujet ou pour préciser les conditions requises pour qu'il y ait recevabilité. On ne peut le voir qu'en soulignant, face au discours individuel, les embarras du discours scientifique comme ceux du discours pénal.

Dans cette perspective, on se demandera s'il existe une ou des criminologies; nous avons nettement indiqué au départ que nous optons pour la deuxième solution ${ }^{12}$. Il importe neanmoins de repartir du discours criminologique type, c'est-à-dire de celui qui met à l'avant-plan l'examen médico-psychologique et social. Pour éviter tout malentendu,

11. Voir Boltanski (1989).

12. Nous avons déjà parlé de «différentes criminologies * (voir Debuyst, 1985b). L'intervention commence de cette manière: «Est-il adéquat de dire qu'il existe des criminologies et non pas une criminologie, ou même, pour être plus précis, des criminologies cliniques et non pas une criminologie clinique? " Notre réponse était oui. 
nous savons fort bien que ce préalable de l'examen médicopsychologique et social s'est particulièrement imposé face à la perspective pénale et à sa référence à une certaine conception du libre arbitre dans la détermination des peines. À ce niveau, nous pourrions comprendre la pretention des criminologues: «avant de prendre une mesure, comprendre». Le problème n'est donc pas là. Il se situe dans le fait que nous opposons un autre discours criminologique à celui que nous pourrions appeler "positiviste" et dans lequel le comportement d'infraction est considéré comme le fait de départ. Si nous le faisons, c'est pour répondre aux «embarras» dans lequel un tel discours nous met. Nous essayerons de le montrer de deux manières: d'une part, en comparant l'examen criminologique à d'autres types d'examen, d'autre part, en indiquant, à travers une brève revue de la documentation, la manière dont le discours du délinquant est disqualifié.

\subsection{L'examen criminologique: répond-il aux exigences liées à la notion d'examen?}

Lorsqu'il s'agit d'un examen médical, le problème paraît à première vue simple: le diagnostic que l'on pose est essentiel dans la détermination du «mal », de ses causes et, par le fait même, dans la mise en place du traitement. Il s'agit d'un constat qui repose sur un certain nombre d'éléments objectifs appréciés comme ayant un rapport causal avec la maladie et que le sujet (ou le patient) est amené à reconnaître et à accepter. Certains peuvent adopter la position qui consiste à dire que l'examen criminologique n'est pas tellement différent de l'examen médical en ce sens que ce qui importe sont les «faits», c'est-à-dire l'infraction, dont le caractère négatif est équivalent à une maladie, ou à un échec scolaire (pour un examen d'orientation scolaire): une sorte d'échec social. On pourrait rechercher dans le contexte situationnel comme dans la personnalité du sujet, ce qui pourrait expliquer cet «échec", en essayant d'établir entre eux une relation de cause à effet. Qu'il s'agisse d'un acte ayant pour le sujet une signification et une justification particulières n'aurait, dans cette perspective, qu'une importance secondaire: ce qui importe est la constatation de ce «mal objectif " que constitue l'infraction et, dans un deuxième temps, le fait de mettre au point un traitement à partir des connaissances recueillies. Nous résumerons cette manière de penser en disant que nous nous trouvons devant une situation à laquelle il importe de réagir: un comportement délinquant, une maladie, un échec scolaire ou une difficulté professionnelle. Tout comme l'examen criminologique constituerait un constat qui porterait sur les aptitudes ou inaptitudes sociales que 
présente le sujet et qui nous explique son comportement. Un tel constat, le sujet devrait l'accepter comme on accepterait n'importe quel diagnostic. En d'autres termes, il importe que le sujet accepte (et qu'éventuellement on le force à accepter) de se laisser considérer comme «objet» d'examen et, par la suite, comme « objet» de traitement. Cette attitude peut paraître réductrice. En réalité, elle est le propre de toute attitude scientifique: dans la mesure où l'on définit celle-ci comme recherche explicative causale, on ne s'intéresse qu'à ce qui est susceptible de faire l'objet d'un raisonnement de cet ordre; c'est à partir de cela que l'on conçoit l'intervention. Ainsi, dans l'examen médical, le patient regarde son corps comme «objet » à propos duquel un traitement doit être mis en place. Dans le cadre d'un examen scolaire ou professionnel, les différentes aptitudes (niveau intellectuel, etc.) que l'on a «mesurées» sont également des éléments objectifs que l'on constate et à partir desquels il importe de décider de l'orientation à suivre. L'examen et le traitement criminologiques pourraient operer de la même manière.

Quelles sont les exigences pour qu'une pareille prise de position puisse se soutenir et quelles sont, de ce fait, les difficultés qui surgissent ? Premièrement, il importe que le sujet accepte d'être transformé en «objet» ou, en tout cas, qu'il soit capable de prendre, par rapport à lui, une distance suffisante pour qu'un aspect de lui-même fasse l'objet d'une telle réduction et qu'il en perçoive l'utilité. Cela se fera sans trop de difficulté en médecine, car généralement, la responsabilité du patient n'est pas personnellement mise en cause par la maladie ou par un traumatisme déterminé qui survient comme un «fait extérieur» (ou alors la mise en cause n'est qu'indirecte: négligences ou imprudences commises). Deuxièmement, le sujet doit avoir confiance aux méthodes utilisées et que, dès lors, les conclusions qui en résultent soient acceptables dans ce qu'elles prescrivent. À ce niveau, nous aurons déjà des difficultés pour ce qui est de l'examen médical et il n'est pas rare que le patient oppose son point de vue ou son interprétation à celle qui lui est proposée. Dernièrement le patient ou le client sait fort bien que le médecin ou le psychologue reste attentif à ses intérêts, et que leur prise en compte interviendra pour une part importante, sinon essentielle, dans la décision. On peut dire que l'examen criminologique ne répond généralement à aucune de ces exigences: d'une part, le sujet est soumis à un examen du fait d'un comportement qui a été voulu et que, bien souvent, il considérait comme une solution plus ou moins justifiée ou comme une forme d'expression et de réalisation de lui-même. Il n'admettra donc le caractère «négatif» du fait que d'une manière limitée ou avec ambivalence. Lorsque les instances pénales (et, à partir d'elles, criminologi- 
ques) ne voient ce comportement qu'à travers son seul aspect d'infraction et que le ton de l'intervention est de type accusatoire, accepter d'être transformé en objet d'investigation ne va pas de soi et sera généralement le résultat d'une pression impérative. Les réponses sont tout aussi négatives ou dubitatives en ce qui concerne les deuxième et troisième exigences.

Il nous paraît donc difficile d'admettre un parallélisme entre ces types d'examenś. Déjà, lorsque nous parlons d'examen médical ou scolaire, la vue que nous en avons donnée reste discutable. Mais, au moins, il était possible de tenir compte de faits objectifs à propos desquels les partenaires étaient capables de s'entendre et de leur donner la même signification. Il était dans cette mesure possible que le sujet s'accepte comme «objet d'examen » et que les résultats de ceux-ci deviennent un élément important dans la politique décisionnelle. Cependant, il pourrait difficilement en être ainsi lorsque les faits sont un comportement délinquant sans que celui-ci soit envisagé dans sa complexité et dans les rapports qu'a le sujet avec les instances décisionnelles. Lorsque nous avons dit, au départ de ce paragraphe, que la règle à toute intervention était de "connaître d'abord la situation avec de décider», on doit en effet se demander ce qu'il importe de «connaître »: sont-ce les caractéristiques de la personnalité «actuelle» du sujet en vue de déterminer le type de traitement? ou les caractéristiques d'un sujet réagissant, à travers et au-delà de l'acte commis, à une situation d'ensemble qui lui est faite et qu'il envisage nécessairement par rapport à d'autres paramètres plus difficiles à exprimer et qui sont rarement pris en compte? Cette modification de perspective ne veut pas nécessairement dire que toute ce qui concerne les caractéristiques de la "personnalité actuelle» soient à éliminer. Elles ont, néanmoins, à être recentrées par rapport à un autre objectif: comprendre les faits à travers une relation que l'École de Lyon appelait sociopathique ${ }^{13}$ et qui implique aussi, d'une manière ou d'une autre, la responsabilité du groupe social.

\subsection{Perspective scientifique et disqualification du discours du délinquant}

Nous avons affaire, ici, au deuxième aspect qu'il importe de voir. 1) Les recherches criminologiques ont, à de nombreuses reprises, fait état du discours du délinquant (ou, plus généralement, du discours du détenu) dans lequel il manifestait ses griefs à l'égard de la société ou les

13. Nous entendons le terme de * relation sociopathique $»$ dans le sens où l'entendent $\mathrm{M}$. Colin et J. Hochmann, c'est-à-dire une relation dans laquelle le sujet - ou les sujets - font l'expérience d'un rejet ou d'une non-communication sociale. 
justifications qu'il cherchait à donner à son comportement. Nous pourrions faire allusion au sentiment d'injustice subie décrit par E. De Greeff (1935) et dont le psychiatre belge faisait une des caracteristiques de le mentalité des récidivistes. De la même manière, les mécanismes de rationalisation ou de justification décrits par Sykes et Matza (1957) constituent également une référence classique. D'une manière moins thérique et pour le confirmer, on voit qu'aux points du questionnaire d'Eysenck, qui se réfèrent à la responsabilité d'autrui dans les ennuis que connaît le sujet, la différence entre délinquants emprisonnés et non-délinquants était nettement significative. Ainsi, à des questions du genre: «Avez-vous eu dans la vie plus de difficultés que la plupart des gens?", un peu plus d'un dixième des non-délinquants répond oui, alors que le pourcentage est près de $50 \%$ pour les délinquants emprisonnés ou soumis à une mesure de défense sociale ${ }^{14}$. À la question: "Auriez-vous mieux fait si les gens ne vous avaient pas mis des bâtons dans les roues?", près d'un quart des non-délinquants répond oui, alors que, parmi les délinquants, ce pourcentage atteint près des trois quarts; et nous pourrions poursuivre.

Que signifient de telles affirmations? Traduisent-elles une situation réelle qui, d'ailleurs, il ne faut pas l'oublier, pourrait fort bien être liée au fait que ces sujets étaient dans une prison ou en institution de défense sociale lorsqu'ils ont répondu au questionnaire? Ou que leur vie fut effectivement une succession d'ennuis? Ou alors, faut-il dire que de telles réponses participent à une certaine pathologie du sujet liée au caractère à la fois égocentrique et revendicateur de la personnalité qui l'amènerait à ne plus voir le tort commis à autrui et à réinterpréter la situation en fonction des torts subis dans un passé récent ou ancien? Il s'agirait, en quelque sorte, d'un prisme qui amènerait le sujet à interpréter la situation d'ensemble par les injustices subies de sorte que les torts causés par l'infraction seraient mis entre parenthèses? Certains parleront alors d'une manière d'interpréter et de raisonner qui serait « délinquante" ou caractériserait une personnalité criminelle, ce qu'à l'heure actuelle certains psychiatres américains appellent «une manière de penser délinquante» (a criminal mind $)^{15}$. Ceci, en soi, n'est pas impossible. Mais, pour reprendre les termes de Boltanski, il s'agirait bien là d'une interprétation (psychologique ou psychiatrique) de type fort qui supprime la possibilité de prendre en compte les éléments de vérité que pourrait contenir le discours individuel (de type faible). 
2) Nous pourrions continuer dans le même registre et noter avec J. Hochmann (1964) que le discours du délinquant, lorsqu'il est condamné et que des contacts avec les cliniciens s'établissent dans le cadre pénitentiaire, pourrait présenter à leur égard une tentative de séduction, ou plus exactement une forme de recours. La condamnation du tribunal au cours d'un cérémonial public représente pour beaucoup une blessure narcissique profonde. Le détenu, écrit J. Hochmann, peut fort bien chercher à susciter, chez le clinicien ou l'intervenant social, une écoute plus compréhensive et faire de lui une instance susceptible de le juger en appel d'une manière moins blessante que ne l'a fait le tribunal. Cette mise en garde ne manque pas d'intérêt et nous ne pouvons pas la négliger. Elle porte néanmoins en elle, dans la mesure où on tend à la généraliser, une interprétation qui transforme le discours du sujet en une stratégie qui vise à minimiser l'importance des faits et à défendre le moi contre les atteintes qui lui ont été portées.

3) De telles analyses sont donc complexes et on ne peut les écarter. On doit dire d'ailleurs que, pour J. Hochmann, celles-ci n'ont de sens que đans une volonté de restaurer le dialogue «sociétal»: pour lui, la prise en compte du caractère public de la condamnation est une condition nécessaire pour que le sujet puisse se comporter ultérieurement (dans sa propre prise en charge) comme acteur social. Une telle position diffère moins qu'on ne pourrait le croire de ce qu'écrivent certains psychiatres belges ${ }^{16}$ amenés à justifier le séjour de mineurs dans une institution de sécurité maximale: il importe non seulement, disent-ils, que ces mineurs reconnaissent avoir commis des actes délinquants, mais aussi acceptent d'être identifiés ou d'être désignés comme délinquants, ce qu'ils sont en réalité pour le «public» qui les entoure. Cela ne signifie pas qu'il faille s'obnubiler sur leur passé, mais il importe, pour le sujet, disent-ils, de prendre ce passé en charge sans quoi l'enfermement, qui est la mesure imposée non seulement par le juge, mais par le groupe social, perdrait son sens et deviendrait incoherent pour le sujet lui-même. Cette prise de position, qui accepte sans discuter la

16. Documents polycopiés présentés au cours d'un colloque organisé par le ministère de la Culture et des Affaire sociales, secteur Protection de la jeunesse, en 1990, sur le thème: Institution publique de Protection de la jeunesse à régimes ouvert et fermé de la Communauté française. 
pénalité donnée, réclame qu'on y réfléchisse ${ }^{17}$. Elle repose sur le fait que l'homme, devant se définir comme être social, ne peut échapper à ce qui constitue le jugement social porté à son égard. Selon eux, c'est de la reconnaissance de ce jugement qu'il importe de partir si l'on veut poursuivre un «traitement ».

Que peut-on dire à ce propos? C'est qu'ici encore on se rabat sur le secteur de l'exécution de la peine (ou, si l'on veut, de l'exécution de la mesure) pour imposer au sujet qu'il se reconnaisse comme «acteur" réel dans sa prise en charge. Nous disons «imposer", parce que la condition semble bien être qu'il accepte le jugement porté sur lui et que l'on voit comme correspondant à sa conduite. Qu'il accepte également l'interpretation que donnent de cette conduite les instances publiques. Et c'est là que se situe justement le problème.

4) Nous dirons qu'on ne peut sans doute pas écarter a priori une interprétation qui tend à pathologiser les dires du sujet ou à les considerer comme tentative de séduction. Mais il n'empêche qu'une attitude de cet ordre représente une disqualification de ces dires, au nom de ce que Boltanski appelait un discours d'un ordre supérieur, c'est-à-dire en l'occurrence un discours "scientifique » qui s'imposerait et annulerait la réalité de ce que le sujet dit pour en faire le symptôme de ce qu'il est. Il faut ajouter que la supériorité du discours «scientifique» va de pair et s'inscrit dans la supériorité du discours "pénal », et de ce fait, qu'on le veuille ou non, le rend nécessairement suspect.

La prise en compte se trouve en plus écartée de ce qui, pour nous, est un élément essentiel: la réaction plus large qui pousse le sujet à refuser l'aspect massif de la responsabilité qui lui est imposée et qui, pour lui, ne répond pas à une réalité effectivement vécue de cette manière ${ }^{18}$. En d'autres termes, il s'agirait d'un refus d'accepter cette attribution dévalorisante qui lui est faite et qui tend à le réduire à n'être

17. On pourrait rappeler que A. M. Favard (1991), au cours de l'enquête faite auprès des cliniciens, montre la difficulté avec laquelle ceux-ci acceptent d'utiliser comme critère de leur diagnostic la notion de "personnalité criminelle» en vue de penser à partir de là un traitement. Elle explique cette réticence par le fait que les cliniciens privilégieraient «une étiologie sociologique de la délinquance au détriment d'une conception centrée sur les caractéristiques inhérentes au sujet », (p. 127). C'est là effectivement une discussion intéressante, mais l'explication donnée nous paraît un peu simple: après ce que nous venons de dire, on en voit déjà les limites.

18. Nous touchons ici a ce point de jonction entre la psychologie criminelie et la psychologie judiciaire. À ce niveau, les deux domaines s'interpénètrent, et il ne faudrait pas l'oublier au nom d'un académisme qui viserait à catégoriser (et à séparer) ces matières. 
qu'un «objet", que ce soit «objet» de punition ou «objet" d'une mesure de traitement ${ }^{19}$. Ce sont là aussi des réalités qu'il n'est pas possible d'éluder au nom des a priori de départ. On ne peut les sousestimer, car elles constituent, pour les sujets, un obstacle à établir une relation dans laquelle les responsabilités pourraient être effectivement prises en charge ${ }^{20}$. Dans la mesure où la grille de lecture qu'impose le droit pénal reste la plus forte, cette question de la responsabilité vécue risque de ne jamais être résolue d'une manière satisfaisante. C'est dans cette orientation, nous semble-t-il, qu'un développement des connaissances criminologiques devrait pouvoir se faire.

\section{LE CARACTĖRE AUTORITAIRE DU DISCOURS PÉNAL COMME OBSTACLE ET LA NÉCESSITÉ D'UN CHANGEMENT DE PARADIGME}

Nous sommes donc renvoyés au caractère de la loi pénale et même à l'aspect symbolique qu'elle prétend assumer. La question qui se pose est celle de savoir si ce type de réponse n'est pas particulièrement rigide et ne traduit pas une forme d'autoritarisme (à la fois réel et symbolique) qui correspond mal a la situation de fait et aux exigences que celle-ci nous pose dans une politique de résolution des conflits. Il en résulterait que, pour ce qui est de la responsabilité vécue, cette réponse de la réaction sociale n'est jamais ou n'est que rarement perçue comme satisfaisante par ceux que la mécanique pénale prend à parti, et il ne nous paraît pas suffisant d'expliquer cette «insatisfaction» par des caractéristiques liées à la personnalité. Nous nous trouvons donc renvoyés à une réflexion sur le droit pénal et sur sa manière de poser la problématique de la délinquance. Dans un article récent, à la fois clair et nuancé, A. Pires (1991) rejoint d'une manière précise les difficultés que nous avons posées. Il nous montre que la réponse à donner est ellemême complexe et qu'en tant que tel le droit pénal ne présente pas une «entité » aussi homogène qu'on pourrait l'imaginer.

19. Nous rejoignons un des constats sur lequel insistent les psychologues sociaux : l'auteur d'un comportement négatif tend à être réduit durablement à cet élément négatif, et, spontanément, un tel élément est considéré par les autres comme faisant partie du noyau central de sa personnalité (Voir Debuyst 1985a, p. 91 et s.).

20. Il s'agirait de rappeler le rapport déjà ancien présenté par F. Ferracuti à la neuvième conférence des directeurs d'instituts de recherches criminologiques (Strasbourg, 1972): perceptions cliniques et psychologiques de la déviance. Des éléments essentiels se trouvent déjà dans ce rapport. 


\subsection{Une vue simpliste du pénal mise en cause}

Le paradigme dans lequel se situe actuellement le droit pénal le présente comme la réponse nécessaire à une violation des droits fondamentaux de la personne dont le respect apparaît comme condition nécessaire à la vie sociale. Dans cette perspective, effectivement, «la punition pénale est un impératif catégorique ou une nécessité sociale $^{21}$ ». (Il nous paraît justifié d'inclure, à côté du terme de punition, celui de "mesure de défense sociale " qui, d'une certaine manière, en constitue l'équivalent). Il en résulte une différence claire entre droit pénal et droit civil: le premier exige, au-delà d'une simple réparation des dommages, un surplus qui se traduit par une punition ou une mesure de défense contre le coupable. À ce point de vue, la spécificité du droit pénal est effectivement défendue, comme l'est d'ailleurs celle de la criminologie qui, lorsqu'elle se définit comme «étude scientifique du comportement et de la personnalité du délinquant », semble lier son sort à la même logique que celle du droit pénal.

Or, on peut dire que, pour le droit pénal comme d'ailleurs pour la criminologie, une telle attitude est difficilement défendable. Ainsi, comme le note $\mathrm{A}$. Pires, des atteintes à la vie, c'est-à-dire à des valeurs fondamentales, peuvent fort bien être saisies de manières différentes: par le pénal, par le civil ou par l'administratif. C'est-à-dire que ce que l'on imagine être la «solution pénale» présente en réalité un caractère hétérogène: le civil et le pénal ne s'opposent pas comme le blanc s'opposerait au noir. On le voit fort bien, d'ailleurs, lorsqu'un auteur comme Acosta parle, dans le champ qui est normalement celui du droit pénal, de la zone grise que constituent les illégalismes privilégiés, c'està-dire une zone dans laquelle, en ce qui concerne le règlement des conflits, les solutions sont d'un autre genre que celles qui jouent ordinairement pour les illégalismes populaires ${ }^{22}$. Face à ces derniers, les mesures, centrees sur les notions de punition et de réadaptation, s'imposent et «sont particulièrement pauvres, stigmatisantes et répressives». La notion de réadaptation, d'ailleurs, comme celle de traitement, suppose qu'il existe entre le délinquant et le non-délinquant une différence

21. Voir Pires $(1991$, p. 51). Nous voyons indiqué "punition», nous pourrions dire de la même manière « mesure de défense sociale» puisque entre la peine au sens où l'entendent les néo-classiques et les mesures de défense sociale des positivistes existe une nette convergence (voir Debuyst, 1976, p. 157 et s.).

22. Par opposition à la zone «blanche " qui serait le domaine ou ont cours les solutions de droit civil et la zone noire dans laquelle les solutions sont pénales. Dans cette zone grise nous aurions les faits qui concernent la santé publique, l'environnement, la santé et la sécuritế au travail, etc. (p. 57). 
faisant du premier quelqu' un pour lequel une «action » est nécessaire en vue de permettre une réintégration sociale, ce qui, comme le souligne A. Pires, donne à la mesure une certaine image d'humanisme. En revanche, lorsqu'il s' agit d'illégalismes privilégiés, la notion de « réadaptation" paraît ne plus avoir de sens: ententes à l'amiable, réparations civiles, etc. ${ }^{23}$ et le sujet «délinquant» joue dès le départ un rôle d'acteur dans leur mise en place. On pourrait dire que les théories de la peine (c'est-à-đire cette spécificité du pénal qui impose un surplus de punition comme manifestation symbolique) sont pensées en fonction des illégalismes populaires, alors que, concrètement, lorsque l'on regarde l'ensemble des illégalismes et qu'on y inclut les illégalismes privilégiés, l'opposition entre le civil et le pénal ne se justifie plus. À ce point de vue, l'analyse d'A. Pires nous parât particulièrement éclairante. On pourrait le dire également pour la criminologie: envisagée dans sa première orientation, elle propose des élaborations qui se font plus particulièrement à partir des illégalismes populaires, c'est-à-dire du droit penal commun pour lequel la politique des peines ou des mesures de défense sociale paraît aller de soi.

Pour résoudre le problème, A. Pires ${ }^{24}$ propose, au niveau du droit pénal, un changement de paradigme: il se manifesterait d'abord d'une manière négative: abandonner l'idée que la punition pénale est un impératif catégorique et une nécessité sociale, récuser l'idée que les mesures qui relèvent exclusivement du droit civil ne conviennent pas au droit criminel. Ensuite, il se manifesterait d'une maniere positive qui introduirait une nouvelle manière de penser les choses: «les droits des personnes doivent être moins vus comme réclamant la main forte du droit criminel que comme valeurs qui nous poussent à mettre en cause l'état et la forme du droit criminel lui-même ${ }^{25}$ ". Nous retrouverons, d'une autre manière, le problème central que nous avons posé au đépart de cet article en décrivant la deuxième orientation possible et qui, effectivement, ne pourrait se développer qu'à l'intérieur d'une perspective qu'A. Pires appelle «un renversement dans la logique de recherche".

23. A. Pires, op. cit. p. 57.

24. A. Pires, op. cit. p. 51.

25. A. Pires, op. cit, p. 71 . A ce point de vue, A. Pires se réfère explicitement à Baratta. 


\subsection{Exemples d'une autre manière de poser le problème, tant du point de vue pénal que criminologique}

La deuxième orientation, en criminologie clinique, que nous avons décrite au départ de cet article, repose sur l'idée suivante: chaque comportement délinquant se situe dans un contexte qui, dans une certaine mesure, détermine son apparition et le sens qu'il est susceptible de prendre, les problèmes qu'il pose directement ou indirectement au groupe social; celui-ci se trouve ainsi (également dans une certaine mesure) renvoyé à ses propres responsabilités. Nous sommes obligés de tenir compte non seulement de l'infraction commise, mais des divers éléments constitutifs de ce contexte. Il ne nous parât plus possible de réfléchir en termes généraux, mais bien en termes de secteurs déterminés dans lesquels un comportement prend place et transgresse un interdit dont la portée dépend également du secteur dans lequel il s'impose. Un vol dans un grand magasin n'est pas la même chose qu'un vol qui se déroule dans le cadre d'une délinquance organisée, non seulement parce qu'il est d'une gravité moindre et qu'il suppose ordinairement un moindre engagement dans la délinquance, mais parce qu'il met en scène, dans le contexte dont il s'agit, des acteurs sociaux différents et que le type de problèmes (qu'on peut appeler sociaux ou societaux) qu'il pose est également différent. De la même manière, une activité de vol commis par un groupe de jeunes vivant dans un bidonville n'est pas la même chose que des activités qui pourraient, de la même manière, être qualifiées de vols pour des jeunes venant d'un autre milieu. Nous aurions donc affaire à des entités qu'il importe de construire adéquatement et qui peuvent faire l'objet d'une étude limitée dont les conclusions ne sont pas nécessairement extensibles aux autres entités. Mais c'est la seule voie qui nous paraît conduire à une compréhension réelle des comportements et à une saisie de la portée qu'il a pour ses auteurs. Pour mieux le percevoir, nous pourrions ainsi prendre quelques exemples.

Reprenons celui des grands magasins ${ }^{26}:$ quels sont les intervenants qui «construisent» ou participent à la «construction» d'une telle

26. Si nous reprenons cet exemple, c'est tout simplement parce que, dans le passé, l'équipe đe recherche du Centre d'étude de la délinquance juvénile a fait sur ce thème une recherche, sans doute limitée, mais intéressante à de nombreux point de vue. Elle fut précédée par plusieurs réunions auxquelles participaient tous les acteurs intervenants (sauf les jeunes délinquants). À l'époque, les représentants du Parquet avaient une attitude de prévention que nous avons appelée intelligente (la prise en compte des problèmes psychologiques et sociaux que présentaient chaque sujet), mais it leur paraissait impossible que toutes les affaires ne soient pas transmises au Parquet par les grands magasins. 
infraction? Évidemment la loi, puisqu'il s'agit d'un comportement que la loi a défini comme infraction (c'est du moins l'interprétation la plus courante). Cette construction «formelle» ayant été posée, on pourrait dire que certains acteurs participent à une construction de fait de l'infraction, en ce sens que, pour des problèmes de rentabilité ou en vue d'une facilitation de la gestion, un certain nombre de dispositions rendent l'infraction plus susceptible de pouvoir exister ou d'être choisie par d'éventuels transgresseurs, et ce, indépendamment des caractéristiques de ces transgresseurs. On peut ainsi dire qu'existent, comme acteurs intervenants, les grands magasins eux-mêmes par leur type de politique: le genre d'interrelations qu'ils créent, ou qu'ils permettent, avec les objets, les personnes (facilités de préhension, etc.); la transformation d'un endroit commercial en un véritable lieu public. On pourrait parler également, d'une manière plus large, de la manière dont sont valorisés, dans l'imaginaire des jeunes, les objets qui y sont vendus et qui déterminent directement ou indirectement son statut (vêtement, disques, etc.); ensuite, les instances qui font en sorte que la réalisation de ce statut ne peut être atteint autrement que par l'acquisition de ces objets, etc. Un constat de ce genre nous amène à dire que la «construction de l'infraction" n'est pas uniquement le fait de la loi; dans la réalité, celle-ci nous renvoie à un comportement qui se déroule dans un contexte précis et qui donne à l' "appréhension du bien d'autrui sans son autorisation» un sens particulier dans lequel on ne peut sousestimer la responsabilité des «propriétaires» et la manière dont ceux-ci organisent la situation en fonction de leurs propres intérêts. On peut difficilement imaginer qu'en rapport avec ces interêts propres il n'y ait pas non plus une responsabilité propre. Nous ne posons le problème que d'une manière très sommaire et ne l'avons fait que pour un seul point: la construction de fait de l'infraction. Nous pourrions aussi voir les éléments intervenants dans la manière dont on "construit» la désignation d'un sujet déterminé comme «voleur » et qui dépend également de la pratique des grands magasins.

Dans le cas du vol dans les grands magasins, on voit fort bien, dans le domaine de la criminologie, ce qui différencierait les deux attitudes

En revanche, le personnel dirigeant des grands magasins était à l'époque attentif à un certain nombre de suggestions qui laissaient voir clairement leur responsabilité dans cette situation particulière: politique de prêts d'objets pouvant être mise au point, etc. Ce travail date des anneses soixante. On peut constater que ce qui paraissait possible à l'époque ne l'est pratiquement plus à l'heure actuelle, du fait même de la situation économique et du durcissement du climat de concurrence. (Voir C. Debuyst, G. Lejour et A. Racine, 1960). 
que nous avons soulignées au départ: la première consisterait à ne pas tenir compte du contexte genéral ni de la plupart des acteurs, si ce n'est d'une manière totalement accessoire. Centrée sur le fait qu'il s'agit d'une infraction, l'infracteur uniquement sera pris en compte. Il peut l'être en faisant, par exemple, l'objet d'une investigation psychologique ou psychosociale en vue de déterminer son «degré d'engagement dans la délinquance " et en permettant, par le fait même, de voir si cela vaut la peine ou non d'intervenir et comment le faire. La seconde attitude consisterait à poser le problème du vol dans les grands magasins (et non un comportement de vol commis par X ou par $Y$ ) comme constituant une entité propre qui doit se comprendre en rapport avec le fait qu'une pluralité d'acteurs-intervenants jouent un rôle en ce qui concerne la «construction" de l'infraction comme à celui de la «construction» du coupable. Non pas pour les «punir», mais comme constituant des « variables" déterminant la manière dont l'interdit est réellement (ou raisonnablement) posé, la manière dont il est vécu et la manière dont il doit réellement être pris en compte. Il faut bien admettre que le sujet, qui se fait appréhender pour le vol commis et qui, dans la suite, fait l'objet d'une sanction ou d'une absence de sanction, est conscient de la situation particulière dans laquelle son acte prend place. Il se heurte à une loi dont le sens est ambigu et, en tout cas, ne fait pas l'expérience d'une interrelation sociale qui serait régie par une certaine forme d'équité.

De la même manière, si nous avions affaire à un groupe de petits chapardeurs qui provient d'un milieu sous-prolétaire, on pourrait effectivement occulter ce qui, dans leur situation, pose effectivement problème au niveau à la fois sociétal, social et psychologique et n'être attentif qu'à ce qui caractérise leur "personnalité actuelle». Ne pas occulter cette situation sociale, c'est en même temps mettre en cause une série d'intervenants qui, à l'un ou l'autre titre, participent à cette situation d'ensemble. Nous savons fort bien qu'il est impossible de prendre en compte la totalité de ces intervenants, mais s'en tenir à ceux qui, jusqu'à preuve du contraire, paraissent raisonnablement «abordables ». Un effort devrait au moins être orienté dans ce sens. Cela constitue un problème de politique et de stratégie. Ce type d'attitude, pour prendre son sens, exige effectivement que l'on modifie les paradigmes du droit pénal dans le sens proposé par A. Pires. De la même manière, dans le cadre d'une criminologie clinique, le fait de resituer l'acte dans un contexte et dans un ensemble où sont concernés, de façon différente, une pluralité de protagonistes pourrait permettre de mieux prendre en compte les responsabilités qui incombent aux uns et aux autres. Ce serait aussi une manière de ne pas donner l'impression au transgresseur 
de faire l'objet d'un coup de force qui le transforme exclusivement en coupable. Dans un texte que nous avons souligné, la psychiatre belge E. De Greeff disait que le sujet ne pourra jamais atteindre sa propre responsabilité si celle du «corps social » n'était pas avoué, d'une manière ou d'une autre.

Donnons un autre exemple tiré des pratiques que l'on pourrait qualifier de «mineures», parce qu'elles portent sur des infractions peu graves qui se situent dans le cadre de la protection de la jeunesse.

Dans un ouvrage récent sur les Nouvelles tendances dans le droit pénal des mineurs ${ }^{27}$, un des protagonistes relate une expérience de médiation menée à Cologne (modèle "die Waage»), en Allemagne, selon une procédure parajudiciaire: pour une série de cas que nous préciserons, le Parquet renvoie le délinquant à un service social privé dont le personnel est à la fois compétent et motivé pour proposer ce genre de solution ${ }^{28}$. Celle-ci repose d'ailleurs sur l'idée, émise dans certaines législations, qu'il y a suppression de peine pour les infractions à la propriété et aux biens si l'auteur répare le dommage avant que les autorités de poursuite n'en aient connaissance ou s'il s'y est obligé par contrat. Certains disent même que, dans ce cas, l'État n'aurait plus le droit de poursuivre. Voyons ce sur quoi l'accent est mis lorsqu'on décrit ce type de démarche: «le moment décisif du règlement de conflits est moins le résultat des conventions de réparation que le processus même de médiation (ou d'accord) auquel l'auteur comme la victime participent activement en retrouvant l'autonomie et la compétence d'action qui dans le procès pénal classique disparaissent » (comme elles disparaissent également dans une procédure du Tribunal de la jeunesse où le juge détermine où se situe le «bien» du mineur).

Pour qu'un tel règlement de conflits puisse se faire, il n'est pas indifférent que le cadre soit une institution parajudiciaire parce qu'audelà de la compétence et des motivations il s'agit d'un négociateur neutre, c'est-à-dire qui n'appartient pas à la Justice ni aux organisations qui défendent les victimes ni à celles qui soutiennent les délinquants. Dans cette négociation, chacun est mis sur un pied d'égalité en ce sens que chacune des deux parties a la possibilité de parler de ses sentiments,

27. Voir Herz (1990).

28. Selon l'auteur, une telle procédure de négociation pourrait toucher 20 a $30 \%$ des affaires qui sont traitées par le Parquet (après avoir exclu les cas dont l'absence de gravité les aurait de toute manière amenées a être classées sans suite) et, parmi cellesci, il y a plus de $80 \%$ dans lesquelles auteurs comme victimes sont d'accord pour s'engager dans une telle procédure. 
de ses difficultés, de ce qu'il éprouve à l'égard de l'autre. Ainsi, chacun a l'impression de pouvoir expliciter son point de vue et, souvent, d'être compris pas l'autre (même s'il ne s'agit pas d'être approuvé). C'est-àdire que celui qui a transgressé la règle n'est pas dès le départ identifié à ses caractéristiques de transgresseur. Il peut être perçu dans ses difficultés ou à partir des injustices antérieures dont il s'est senti victime. Cette participation active de l'auteur et de la victime semble montrer qu'à l'intérieur d'une telle procédure «le besoin supposé de sanction de la part de la population relève plutôt d'une fiction des juristes et que la victime se montre volontiers compréhensive».

En conclusion, ce qu'il nous paraît important de noter, c'est qu'une procédure de ce genre ne constitue pas essentiellement une «expérience de responsabilisation » du jeune; elle constitue une expérience d'équité pour les protagonistes. La responsabilisation viendra après et ne peut que se greffer sur une expérience de ce genre. Expérience d'équité, parce que la situation de l'un et l'autre est prise en compte et que ce qui en est dit est reconnu par tous comme ayant quelque chose de « raisonnable» ou d'acceptable. Et s'il en est ainsi, c'est parce que chacun voit que son droit à la parole n'est pas dénaturé par des a priori de départ.

Nous nous trouvons donc fort proches de la position que décrit Habermas (1986) dans Éthique et Communication et où il cherche à établir des règles pour qu'existe «une mise à profit équitable de ses droits ${ }^{29}$ ». Nous ne donnons ici qu'une partie des règles qu'il propose: «Tout sujet capable de parler et d'agir doit pouvoir prendre part à des discussions. Chacun doit pouvoir problématiser toute affirmation, quelle qu'elle soit. Chacun doit pouvoir admettre dans la discussion toute affirmation, quelle qu'elle soit. Chacun doit pouvoir exprimer ses points de vue, ses désirs et ses droits. Aucun interlocuteur ne doit être empêché par une pression autoritaire, qu'elle s'exerce à l'intérieur ou à l'extérieur de la discussion, de mettre à profit ses droits tels qu'ils sont établis dans les points précédents» (p. 110-111). Ce seul aspect nous permet déjà de comprendre ce que pourrait signifier ce renversement de la logique à la fois de communication et de recherche à laquelle faisait allusion A. Pires.

\section{CONCLUSION}

Nous clôturerons ces quelques notes par trois remarques d'ordre psychologique. Nous nous contenterons de les présenter d'une manière

29. Ces propositions sont, en fait, émises par Alexy et reprises par Habermas. 
rapide $^{30}$; elles nous paraissent néanmoins mettre l'accent sur un point important qui en plus semble être, à l'heure actuelle, un point sensible: la «responsabilisation» du sujet. Ces quelques remarques visent a insister sur le fait que cette «responsabilisation" doit nous renvoyer à un processus actif qui presente une tonalité intellectuelle autant qu'affective. Cela signifie que le sujet doit pouvoir participer à la manière de définir la situation qui le concerne. Cela n'est possible que dans la mesure où son point de vue est pris en compte et qu'est prise en compte également la responsabilité des autres acteurs éventuels. C'est, semble$\mathrm{t}$-il, une condition nécessaire que le sujet puisse effectivement « naître » à sa propre responsabilité ${ }^{31}$.

Une première remarque vise à mettre l'accent sur les mécanismes de centration et de décentration qui caractérisent les attitudes des protagonistes dans le cadre d'un conflit et sur lesquels des auteurs comme J. Piaget et $\mathbf{W}$. Doise ont insiste $e^{32}$. Le problème réside dans le fait que chacun risque de rester centré sur son point de vue et devient de ce fait totalement incapable d'intégrer dans son optique les éléments du point de vue adverse dont le bien-fondé est néanmoins réel. Cette difficulté vaut sans doute pour le délinquant, mais aussi pour son vis-à-vis, le juge ou, plus largement, l'opinion publique. La difficulté consiste donc à établir de nouveaux liens entre les éléments jugés opposés et constitutifs de la situation vécue par les deux sujets et dans lesquelles ils se trouvent ancrés. Il s'agit d'une véritable «invention" de l'intelligence ou, dans ce cas, dirions-nous, d'une invention éthique. Il s'agirait donc de créer des conditions requises pour qu'une telle dynamique puisse se constituer, et c'est ce que nous trouvons, dans une certaine mesure, dans l'expérience de médiation «Die Waage» que nous avons citée au début de ce paragraphe.

Nous dirons dans une deuxième remarque que, dès les entretiens que nous avions menés, aux alentours des années soixante, à la Prison centrale de Louvain avec des jeunes détenus ayant commis cette fois des faits graves, la plupart des sujets mettaient violemment l'accent sur les torts dont ils avaient été victimes dans le présent et le passé et

30. Voir Debuyst (1989).

31. On ne peut pas dire qu'il s'agisse ici d'un problème social ou sociologique auquel on nous a souvent reproché de réduire la clinique. Il s'agit d'un problème essentiellement psychologique.

32. Voir particulièrement Piaget (1975), W. Doise et Mugny (1981), ainsi qu'un article manuscrit de Doise: la double dynamique sociale dans le développement cognitif, Université de Genève, (1990). 
montraient de ce fait un sentiment marqué d'injustice subie ${ }^{33}$. Mais, dans un deuxième temps, il leur devenait possible d'accepter le fait que par leur comportement ils étaient eux-mêmes responsables de torts graves à l'égard de personnes connues ou inconnues. En d'autres termes, tout se passe comme s'il ne leur était possible d'accepter cette évidence qu'à partir du moment où ils avaient l'impression d'avoir été entendus dans leur propres affirmations et s'étaient sentis perçus comme interlocuteurs, c'est-à-dire comme quelqu'un dont la perspective propre n'etait pas forcément disqualifiée par une autre perspective que l'on pourrait qualifier de "officielle» et qui leur était imposée. $\mathrm{Si}$ rien n'est résolu par une nouvelle relation de ce genre, il pourrait s'agir néanmoins d'une orientation de départ qui, d'un point de vue théorique, nous parait congruente avec d'autres observations.

$\mathrm{Et}$, finalement, troisième remarque, des initiatives prises sous l'impulsion du psychologue américain Kohlberg et qui visent à créer, dans le cadre institutionnel pour jeunes délinquants, ce qu'il appelait une "communauté juste ${ }^{34}$ constituerait également un type d'expérience à analyser. Nous voyons donc, et ce seront nos dernières conclusions, que ces trois remarques supposent d'une manière très précise une modification des paradigmes, tant dans le domaine pénal que dans celui de la criminologie. Cela n'entraîne pas un rejet de tout un acquis préalable que l'on ne peut pas mésestimer (et nous l'avons vu tout au cours de ces notes), mais un changement de perspective dans le statut qu'occupe cet acquis et dans la manière de le resituer.

\section{BIBLIOGRAPHIE}

BOLTANSKI, L. (1989), L'amour et la justice comme compétences, Paris, Métaillé.

CAIRO, R., FAVARD, A.-M. (sous la direction de) (1991), La personnalité criminelle, Toulouse, Érès.

DEBUYST, C. (1991), «Les choix faits en criminologie et la manière dont ils se trouvent insérés dans le cadre institutionnel ", in L'intervention psychosociale au sein du pénal, Actes de la $1^{\text {èr }}$ Conférence internationale du GREPO, département de criminologie, Université d'Ottawa.

DEBUYST, C. (1989), «Criminologie clinique et inventaire de personnalité : utilisation quantitative ou qualitative », Déviance et Société, vol. 13, n 1, p. 1-21.

33. Voir Debuyst (1960).

34. Voir Kohlberg et collab., (1975). Cité par J. Dionne dans son document de travail préparatoire au doctorat et déposé au département d'éducation, Université McGill, Montréal (1987). 
DEBUYST, C. (1989), «Perspectives cliniques en criminologie. Le choix d'une orientation», Revue internationale de criminologie et de police technique, vol. 4, p. $405-418$.

DEBUYST, C. (1985a), Modelle éthologique et criminologie, Bruxelles, Mardaga éditeur.

DEBUYST, C. (1985b), « Le droit pénal et les différentes problématiques possibles en criminologie», in La criminologie au prétoire, (p. 165-180), Bruxelles, StoryScientia.

DEBUYST, C. (1976), «Les conceptions criminologiques de la culpabilité », Annales de l'Université des sciences sociales de Toulouse, vol. XXIV, $\left(\mathrm{n}^{\mathrm{os}} 1\right.$ et 2$), \mathrm{p} .151-$ 172.

DEBUYST, C. (1960), Criminels et valeurs vécues, Louvain et Paris, Nauwelaerts.

DEBUYST, C., LEJOUR, G., RACINE, A. (1960), Petits voleurs de grands magasins, Bruxelles, CEDJ.

DE GREEF, E. (1935), «Le sentiment d'injustice subie en pathologie criminelle», Annales médico-psychologiques, vol. 3, p. 24.

DOISE, W., MUGNY, G. (1981), Le développement social de l'intelligence, Paris, Interedition.

FAVARD, A.-M. (1991), «Personnalitê criminelle: de la validation à la validité», in R. Cario et A.-M. Favard (sous la direction de), La personnalite criminelle, Toulouse, Erès.

FERRI, E. (1905), La sociologie criminelle, (trad., française), Paris, F. Alcan.

HABERMAS, J. (1986), Morale et communication, Paris, Édition du Cerf.

HERZ, R. G. (1990), «Médiation entre auteur et victime: le modele die Waage" a Cologne», in F. Dünkel et J. Zermatten, Nouvelles tendances dans le droit pénal des mineurs, Freiburg, rapports criminologiques de l'Institut Max Planck de Droit Pénal Étranger et International, tome $\mathbf{4 2 .}$

HOCHMANN, J. (1964), La relation clinique en milieu pénitentiaire, Paris, Masson.

KOHLBERG, V., et al. (1975), «The just community approach to correction: a theory \#, Journal of Moral Education, vol. 4, n 3, p. 243-260.

PIAGET, J. (1975), L'équilibration des structures cognitives, Paris, PUF.

PINATEL, J. (1963), Criminologie, (tome 3 du *traité de Droit pénal et de criminologie» de P. Bouzat et J. Pinatel), Paris, Dalloz.

PIRES, A. P. (1991), «Ethiques et réforme du droit criminel: au-dela des philosophies de la peine », Ethica, vol. 3, $n^{\circ} 2$, p. 47-78.

SYKES, G. H., MATZA, D., (1957), «Techniques of neutralization. A theory of delinquency ", American Sociological Review, vol. 22, p. 664-670.

WALTERS, V., WHITE, T. (1989), "The thinking criminal. A cognitive model of life style criminality $»$, Criminal Justice Research Bulletin, $n^{\circ} 4$. 